Hemodynamics and progressi on of a hypervascul ar focus i $n$ a bor derline I esi on of hepat ocel I ul ar car ci noma : anal ysi s by angi ogr aphy- assi st ed CT and hi st opat hol ogy

\begin{tabular}{|c|c|}
\hline 著者 & 遠藤 珠生 \\
\hline 著者別表示 & Endo Tamao \\
\hline $\begin{array}{l}\text { jour nal or } \\
\text { publ i cat } i \text { on } t i t l e\end{array}$ & 博士論文本文Ful I \\
\hline 学位授与番号 & 13301甲第3994号 \\
\hline 学位名 & 博士 (医学) \\
\hline 学位授与年月日 & $2014-03-22$ \\
\hline URL & ht t p: //hdl . handl e. net /2297/40462 \\
\hline
\end{tabular}




\title{
Hemodynamics and progression of a hypervascular focus in a borderline lesion of hepatocellular carcinoma: analysis by angiography-assisted CT and histopathology
}

\author{
Tamao Endo $\cdot$ Kazuto Kozaka $\cdot$ Satoshi Kobayashi $\cdot$ Junichiro Sanada \\ Wataru Koda · Tetsuya Minami - Azusa Kitao • Norihide Yoneda • \\ Yasuni Nakanuma · Toshifumi Gabata · Osamu Matsui
}

Received: 3 October 2013/Accepted: 13 November 2013/Published online: 12 December 2013

(C) Japan Radiological Society 2013

\begin{abstract}
Purpose To investigate the hemodynamics and progression of a hypervascular focus (HF) in a borderline lesion by dual-phase CT during hepatic arteriography (CTHA) and reveal the process of the transformation to hypervascular overt hepatocellular carcinoma (HCC).

Materials and methods This study was performed with the approval of our institutional ethics committee, and informed consent for the retrospective usage of clinical materials was obtained from all the patients. The 121 nodules in 76 consecutive patients with liver cirrhosis and chronic hepatitis showing an HF in a borderline lesion on angiography-assisted CT were analyzed. Hemodynamic changes were observed in 24 patients who underwent repeated angiography-assisted CT. Histopathological analysis was conducted in eight nodules.

Results HF was classifiable into type A (stain disappeared), B (stain prolonged), C (stain was washed out and corona-like drainage into the outer nodule was seen) and D

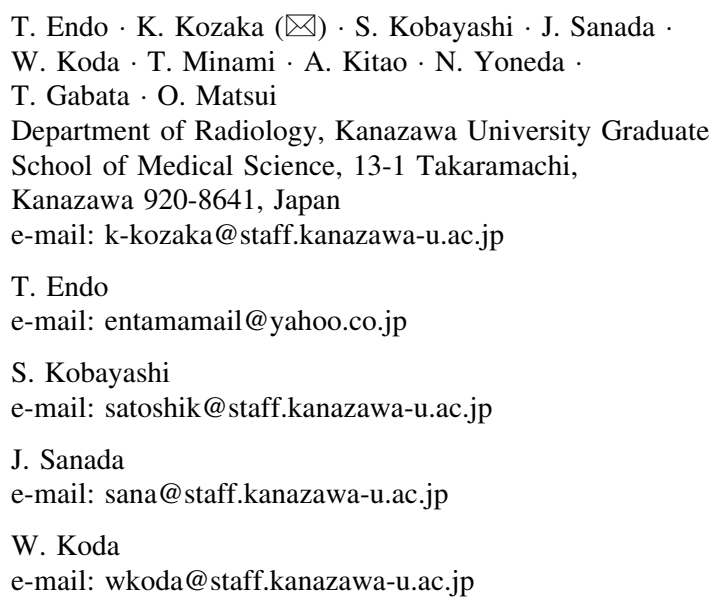

(stain was washed out and corona-like drainage into the whole outer nodule was seen) on the late phase of CTHA and was seen to progress in this order on follow-up observation. Histopathologically, de-differentiated foci showed significantly higher expression of sinusoidal capillarization and unpaired arteries than background nodules and showed pseudocapsule, compressive and replacing growth at the border of the background nodule.

Conclusion HF showed multi-step progression and transformation to hypervascular overt HCC.

Keywords Hepatocellular carcinoma .

Multidetector computed tomography · Angiography ·

Precancerous conditions - Neovascularization .

Pathologic

\section{Abbreviations \\ HF Hypervascular focus \\ CTHA CT during hepatic arteriography}

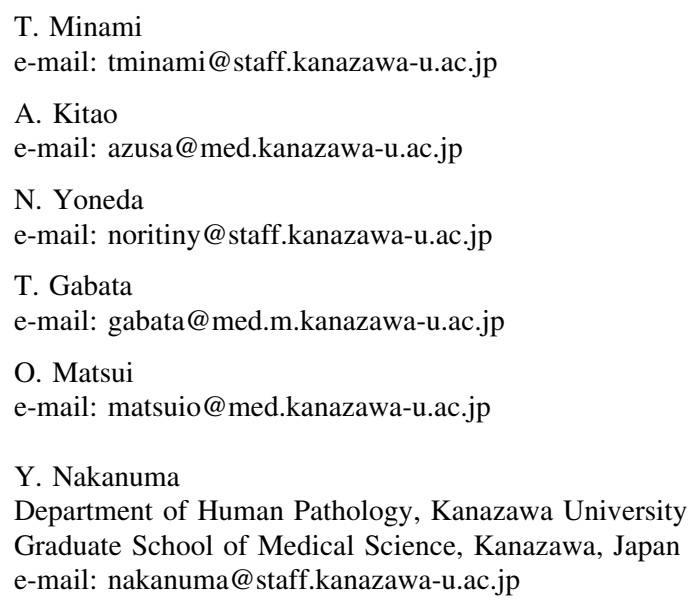




\section{HCC Hepatocellular carcinoma}

DN Dysplastic nodule

CTAP CT during arterial portography

\section{Introduction}

Hepatocellular carcinoma (HCC) is one of the most common cancers worldwide and one of the most common causes of death in many countries [1]. To improve the outcome of patients with HCC, the precise detection and characterization of early stage HCC by imaging and the most appropriate treatment based on an understanding of the pathophysiology of HCCs are essential.

Chronic infection of hepatitis B and/or C virus is well known to be the most common etiology of HCC, and chronic inflammation due to these infections evokes genetic alterations resulting in hepatocarcinogenesis [2]. In this situation, HCC commonly shows multi-step hepatocarcinogenesis from dysplastic nodule (DN), early HCC and well differentiated HCC to moderately or poorly differentiated HCC [3]. Because the precise differentiation between DN and early HCC is not definitely possible by conventional imaging diagnosis and/or biopsy, these are often called "borderline lesions" in clinical practice. During this multi-step hepatocarcinogenesis, neoangiogenesis plays an important role in the progression of the degree of malignancy of the nodules [4].

It has been verified that, during multistep hepatocarcinogenesis, a hypervascular de-differentiated HCC component appears in a hypovascular borderline lesion [3, 5-8]. This focus grows to finally occupy the entire nodule, resulting in progression to a more malignant and entirely hypervascular HCC (so-called classic HCC) [8]. In addition, in accordance with the progression of these changes, the drainage vessels change from hepatic veins in borderline lesions to hepatic sinusoids and then to portal veins in hypervascular classic HCCs [9]. Angiography-assisted CT, including CT during arterial portography (CTAP), CT during hepatic arteriography (CTHA) and single-level dynamic CTHA, is well known to be the most accurate modality to evaluate these hemodynamic changes during hepatocarcinogenesis [6,7].

Investigating the hemodynamics of a hypervascular focus (HF) in borderline lesions and the changes occurring with the progression of hepatocarcinogenesis is important to understand the imaging findings and pathophysiology of early stage HCC. The purpose of this study was to evaluate the hemodynamics and their longitudinal changes in an HF in a borderline lesion by evaluating repeatedly performed CTHA images and to clarify the process of the transformation from a hypovascular borderline lesion to entirely hypervascular classic HCC.

\section{Materials and methods}

This retrospective study was performed with the approval of the institutional ethics committee, and informed consent for the retrospective usage of clinical materials was obtained from all the patients.

Definition of a HF in borderline lesions

In imaging analysis, a "borderline lesion" was defined as a nodule showing hypodensity on CTHA indicating decreased intranodular arterial supply and isodensity or slight hypodensity on CTAP indicating the same or decreased but not absent intranodular portal supply relative to the surrounding liver parenchyma [7, 8]. When early phase of a CTHA image indicated a region of hyperdensity relative to the surrounding area in the nodule and its largest diameter was not larger than half of the largest diameter of the entire borderline lesion measured on axial images, it was defined as an HF [7, 8]. Finally, a borderline lesion that contained an HF was defined as a "borderline lesion with an HF."

It is well known that an HF within a hypovascular borderline lesion on imaging diagnosis corresponds to a more de-differentiated HCC portion in a DN or hypovascular early HCC histologically $[3,4,7]$.

\section{Patients}

For imaging analysis, 121 nodules in 76 consecutive patients with liver cirrhosis and chronic hepatitis showing an HF in a borderline lesion on CTHA and CTAP between January 2000 and October 2010 were included in the study. The patients comprised 45 men and 31 women aged 49 to 85 years (mean age, 68 years). Details of the background livers were as follows: hepatitis $\mathrm{C}$ viral-related cirrhosis and chronic hepatitis, 63; hepatitis B viral-related cirrhosis and chronic hepatitis, 10; cirrhosis and chronic hepatitis with unknown etiology, 3. Hepatic function was classified as Child-Pugh class A in 55 patients, class B in 20 patients and class $C$ in 1 patient (Table 1).

Both CTAP and CTHA were performed in all 76 patients for the precise staging of HCC before radiofrequency ablation, surgical resection or superselective (subsegmental) transcatheter chemoembolization (TACE). Thirty-nine hypervascular foci in borderline nodules in 24 patients, who were treated with repeated TACE for other hypervascular classic HCCs in different segments, had the chance for repeated CTAP and CTHA examination without any local treatment. The mean periods between the repeated CTHA and CTAP were 383 days (range 40-855 days) in these patients. In addition to CTAP and CTHA, singlelevel dynamic CTHA examination was performed in 20 
Table 1 Baseline characteristics of 76 patients

\begin{tabular}{ll}
\hline Characteristic & Value \\
\hline Median age (years \pm SD) & $68 \pm 9$ (range 49-85) \\
Sex (male/female) & $45 / 31$ \\
Child-Pugh classification (A/B/C) & $55 / 20 / 1$ \\
Etiology and histology of underlying liver disease \\
HCV-related cirrhosis & 47 \\
HCV-related chronic hepatitis & 5 \\
HBV-related cirrhosis & 6 \\
HBV-related chronic hepatitis & 2 \\
Cryptogenic cirrhosis & 1 \\
Cryptogenic hepatitis & 2
\end{tabular}

Histopathological specimens were not available in $11 \mathrm{HCV}$-related and $2 \mathrm{HBV}$-related hepatitis patients

$S D$ standard deviation, $H C V$ hepatitis $\mathrm{C}$ virus, $H B V$ hepatitis $\mathrm{B}$ virus

nodules in 17 patients for the differential diagnosis between hypervascular pseudolesion and HCC [10, 11].

For the histological diagnosis and analysis, biopsy specimens were not employed in this study because it was impossible to evaluate the entire nodule. Among all 121 nodules, 8 were surgically resected and were subjected to the histological analysis. The flow diagram of this study is provided in Fig. 1.

Angiography-assisted CT examination

CTAP, CTHA and single-level dynamic CTHA were performed with various CT scanners (XVision SP, Toshiba Medical Systems, Tokyo, Japan; Aquilion 64, Toshiba
Medical Systems; HiSpeed Advantage, GE Healthcare, Milwaukee, WI; LightSpeed Ultra 16, GE Healthcare). From the femoral artery, 4-F catheters were inserted into the superior mesenteric artery for CTAP and into the common, proper or replaced right hepatic artery for CTHA. CTAP scans were obtained at 5-7-mm-section thickness and 5-7$\mathrm{mm}$ collimation to cover the entire liver in a single breath hold. To increase the blood flow and decrease the laminar flow of the portal vein, $5 \mu \mathrm{g}$ of prostaglandin E1 (Palux; Taisho, Tokyo, Japan) was injected into the superior mesenteric artery before contrast medium infusion. Helical scanning began $25 \mathrm{~s}$ after the infusion of $50-70 \mathrm{ml}$ of iohexol (320-350 mg of iodine per milliliter) (Omnipaque; Daiichi, Tokyo, Japan) at a rate of $1.8 \mathrm{ml} / \mathrm{s}$ using a power injector had been started. CTHA scans were obtained at 3-5$\mathrm{mm}$ section thickness and 3-5-mm collimation. Helical scanning was started $7 \mathrm{~s}$ after the beginning of an infusion of iohexol (320-350 mg of iodine per milliliter) into the common, proper or replaced hepatic artery at a rate of 1.0-1.8 ml/ $\mathrm{s}$ (early phase). The infusion of contrast material was continued until $5 \mathrm{~s}$ after the early phase CTHA scanning had been completed. The scanning time varied according to the individual liver size. Thirty seconds after contrast material infusion had finished, the late phase scanning commenced.

Single-level dynamic CTHA was performed with the infusion of $10-18 \mathrm{ml}$ of iohexol at a rate of $1.0-1.8 \mathrm{ml} / \mathrm{s}$ in the common, proper or replaced right hepatic artery using a power injector. Scanning began immediately before the injection of contrast medium. As a minimum, $40 \mathrm{~s}$ continuous scanning was done without table feed (later phase, late half of the sequential scanning). One image per second was reconstructed [10].
Fig. 1 Flow diagram of study sample. $R F A$ radiofrequency ablation, TACE transarterial chemoembolization, pts patients

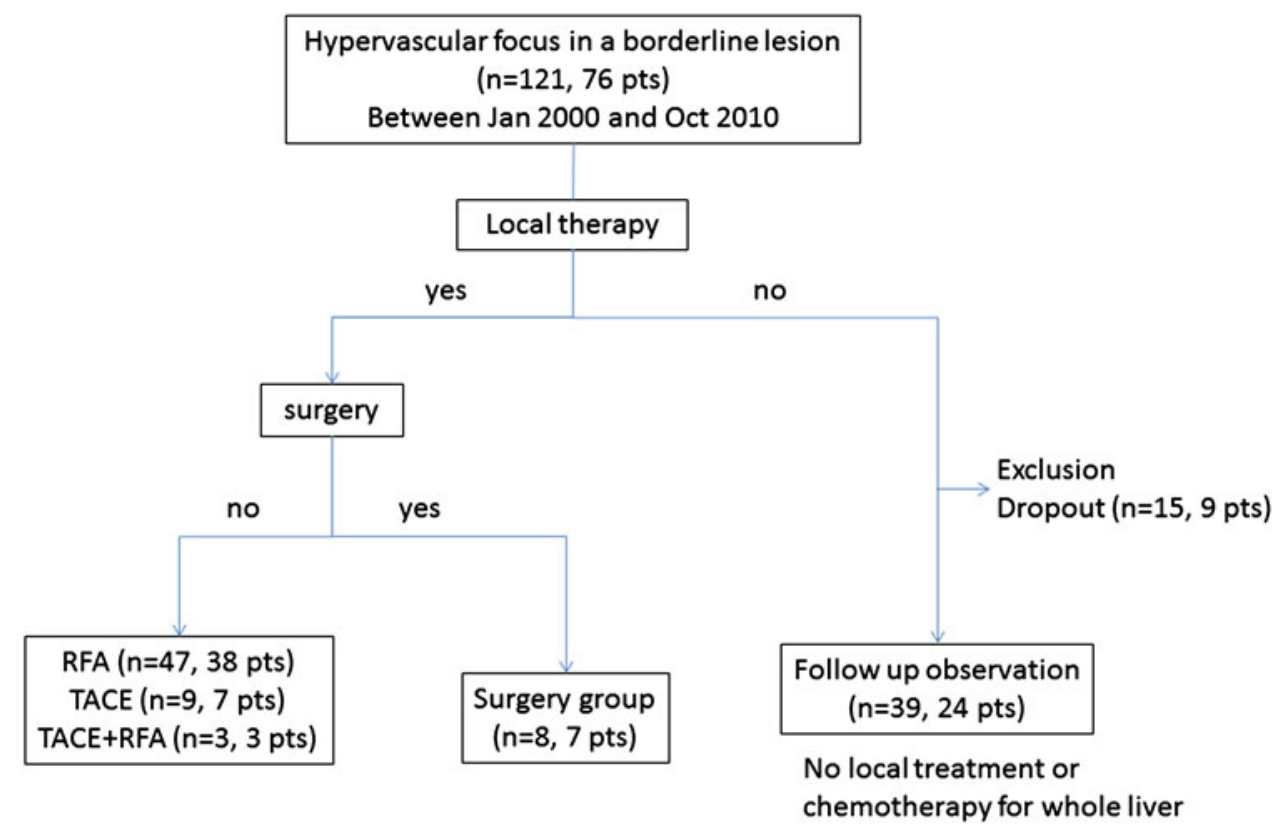



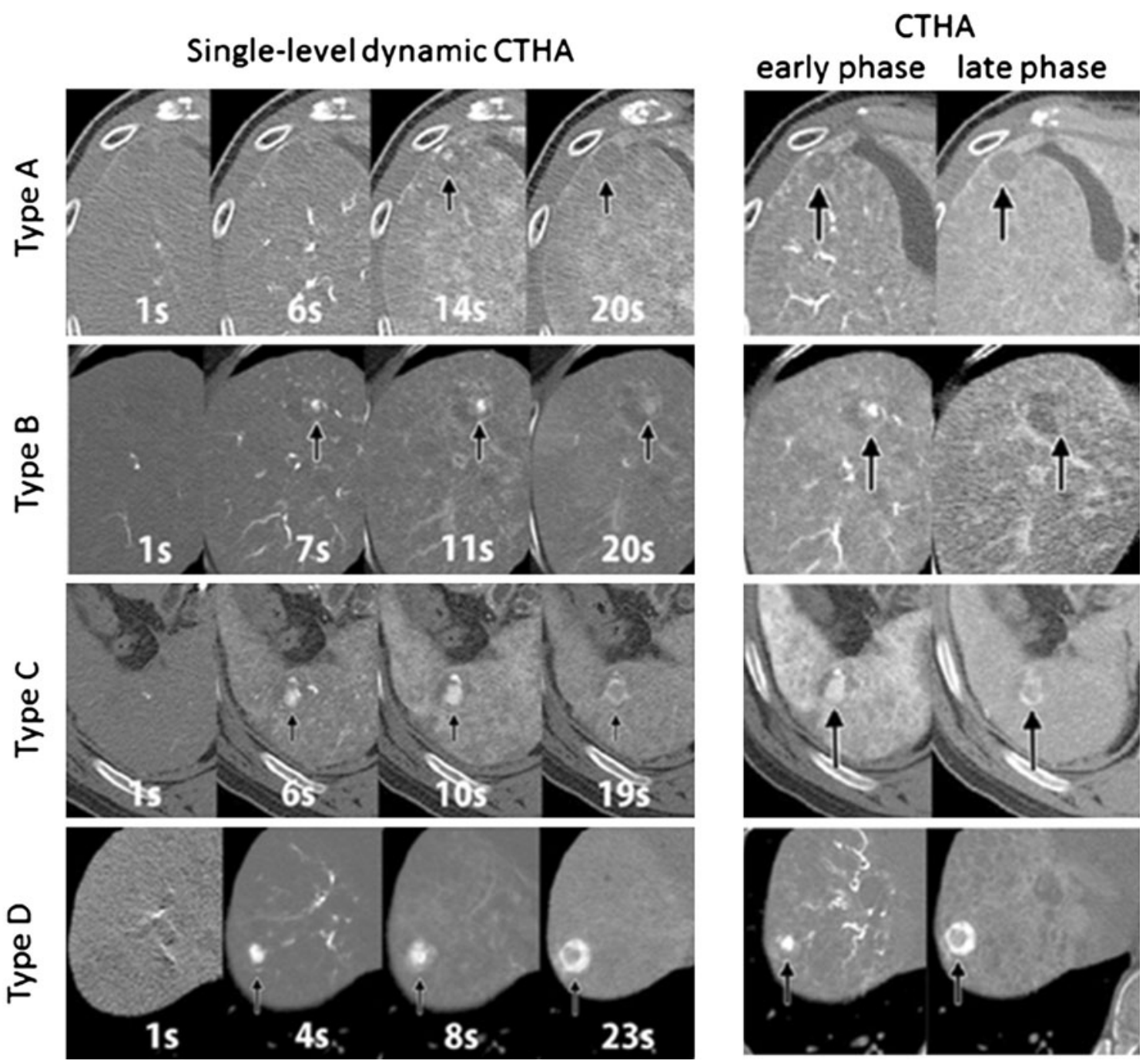

Fig. 2 Classification of hemodynamic patterns of an HF in the borderline lesion based on the findings of single-level dynamic CT during hepatic arteriography (CTHA). In type A, the early staining disappeared gradually and became isodense relative to the surrounding borderline lesion on the later phase. In type B, the early staining enlarged but weakened in density gradually and became a faintly enhanced area in the borderline lesion on the later phase. In type $\mathrm{C}$, the early staining disappeared earlier (washout), and thin coronal enhancement was demonstrated around the early stained area on the later phase. In type D, the early focal staining disappeared earlier (washout), and almost the entire borderline lesion showed diffuse staining on the later phase. Late phase image of CTHA (right) roughly corresponds to that in the later phase of single-level CTHA. $S$ Seconds after the start of contrast injection into the hepatic artery

\section{Image evaluation}

By single-level dynamic CTHA, in vivo hemodynamics in an $\mathrm{HF}$ was evaluated. Using this method, the drainage pattern of the arterial blood flow into the hypervascular lesion can be visualized in real time. According to the previous reports [9, 10], the arterial blood supply into a classical HCC drains into the surrounding hepatic sinusoids or portal venules through the numerous communications between tumor blood sinusoids and hepatic sinusoids and/or portal venules surrounding the tumor, resulting in a ring or corona-like perilesional enhancement at the later phase of single-level dynamic CTHA [9].However, in hypovascular borderline lesions including early HCCs, the main blood supply from the portal vein drains directly into the hepatic veins, and therefore no peri-lesional enhancement is demonstrated. The later phase of single-level dynamic CTHA roughly corresponds to the late phase of CTHA, and therefore the drainage pattern from the hypervascular lesion can also be evaluated on the late phase of CTHA $[11,12]$.

To clarify the hemodynamics and progression of hypervascular foci, the hemodynamic patterns observed by single-level dynamic CTHA were evaluated, and the correlation between the hemodynamic patterns and sizes of the lesions were analyzed. In addition, hemodynamic changes during follow-up periods were analyzed. 
All images were interpreted retrospectively by three experienced abdominal radiologists (T.E., K.K. and O.M., with 13, 13 and more than 20 years' experience, respectively), and all images were analyzed subjectively and independently by them. Disagreements were resolved by consensus.

\section{Pathologic examinations}

Liver specimens were fixed with neutral formalin, and 4-mm-thick paraffin-embedded tissue slices were prepared from each nodule. Hematoxylin \& eosin staining was performed. To clearly differentiate each vessel, double immunostaining with CD34 (a vascular endothelial cell marker) and $\alpha$-smooth muscle actin (a smooth muscle cell marker) was performed in all nodules. Two radiologists (K.K. and A.K., with 13 and 11 years' experience as radiologists, respectively, and both with 3 years' experience in pathological research at the pathology department) and one specialized pathologist (Y.N., 40 years' experience) who were blinded to all imaging information, evaluated histological specimens in consensus.

Tumor differentiation was diagnosed according to the classifications proposed by the International Working Party [3]. Growth patterns of de-differentiated foci were classified into two types: replacing and compressing. In the nodules with replacing growth, tumor cells proliferated by replacing surrounding borderline cells, and the margin between a de-differentiated focus and background borderline lesion was indistinct. Compressing-growth nodules had a discrete margin and pushed out the surrounding borderline cells. In addition, we counted the numbers of vessels (hepatic and neovascularized unpaired arteries, portal veins and hepatic veins) twice within a de-differentiated focus and in the surrounding borderline lesion. Vessels were counted in the largest square we could draw in the respective areas of the tissue slices. Then, we calculated the microvessel density (MVD) by dividing the number of vessels by the area to calculate vessel density (per square millimeter). We also measured the area of capillarized sinusoids covered by endothelial cells expressing CD34 and compared it with the whole area of the nodule in the same slice. The capillarized area in each nodule was semiquantitatively rated as follows: $1+=0-25 \%, 2+=26-50 \%, 3+=51-75 \%$ and $4+=76-100 \%[9]$.

\section{Statistics}

The data were expressed as the mean \pm standard deviation (SD). Statistical significance was evaluated with GraphPad Prism5 (Graph-Pad Software, San Diego, CA, USA). The unpaired $t$ test was used for the analysis of clinical features.
Data from different groups were compared using a one-way analysis of variance (ANOVA) with post test for linear trend. The Kruskal-Wallis test was used for the comparison of the proportion of HF size against the entire nodule size with each type and the comparison of tumor differentiations with those in the hypervascular and hypovascular areas. The results were considered significant if $p$ value was $<0.05$.

\section{Results}

Image analysis

Hemodynamic patterns of an HF in the borderline lesion

On the basis of observation of single-level dynamic CTHA, hypervascular foci in the borderline lesions were classified into four types as shown in Fig. 2. In type A, the early focal staining disappeared gradually and became isodense relative to the surrounding hypodense borderline lesion on the
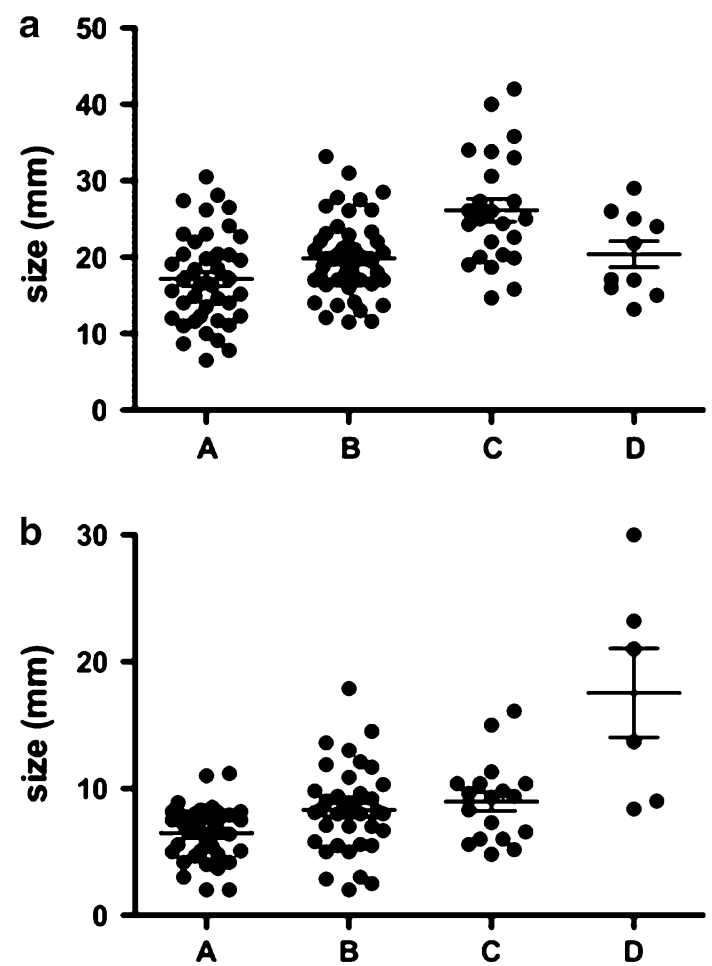

Fig. 3 The correlation between the sizes of the entire borderline nodule and inner HF and each type of hemodynamics of the inner HF. a The sizes (the largest diameter on axial images of early phase of CTHA) of the entire borderline nodule showed a positive relation in order from type A to $\mathrm{D}\left(R^{2}=0.04128, p=0.01\right.$, post test for linear trend). b The sizes (the largest diameter on axial images of early phase of CTHA) of the inner HF showed a moderate relation in order from type A to $\mathrm{D}\left(R^{2}=0.1039, p=0.0002\right.$, post test for linear trend) 
later phase. In type $\mathrm{B}$, the early focal staining enlarged but weakened in density gradually and became a faintly enhanced area with a vague margin in the hypodense area surrounding the borderline lesion on the later phase. In type $\mathrm{C}$, the early focal staining disappeared earlier (washout), and thin coronal enhancement was demonstrated around the early stained area in the hypodense borderline lesion on the later phase. In type D, the early focal staining disappeared earlier (washout), and almost the entire borderline lesion except for the HF showed staining on the later phase. On CTAP, only the HF showed decreased or absent portal blood supply relative to the surrounding borderline lesion in type $\mathrm{A}, \mathrm{B}$ and $\mathrm{C}$ nodules, while almost the entire nodule demonstrated decreased or absent portal perfusion in type D nodules.

These types were applied to the hemodynamic classification of the nodules in which single-level dynamic CTHA was not carried out based on the findings of the early and late phase of CTHA. Of 121 nodules, 42 were classified as type A, 45 as type B, 24 as type C and 10 as type D. The sizes (largest diameter on axial images) of the entire nodules of each group were $17.1 \pm 5.9, \quad 19.8 \pm 5.2$, $26.1 \pm 7.3$ and $20.4 \pm 5.4$, respectively, and the sizes of the internal HFs of each group were $6.0 \pm 2.2,7.9 \pm 2.7$, $9.3 \pm 3.6$ and $9.5 \pm 2.7$, respectively. Both entire nodule size and focus size showed a positive correlation among each A to D group in this order (Fig. 3). The proportion of hypervascular foci size against the entire nodule size was significantly different between each type $(p<0.05)$.

\section{The progression and hemodynamic changes of an HF}

A total of 39 nodules were subjected to repeated CTAP and CTHA examinations without any local treatment. For the purpose of this analysis, we added another type of hemodynamic pattern, namely type E, which demonstrated hyperdensity on the early phase in over half of the entire nodule with ring-like or coronal enhancement on the late phase of CTHA, indicating a classic hypervascular HCC. The progression and hemodynamic changes of an $\mathrm{HF}$ in borderline lesions during the follow-up period are summarized in Fig. 4. The progression and sequential changes of the hemodynamic patterns in hypervascular foci were from type A to type $\mathrm{E}$ in order in all nodules. Figures 5 and 6 shows two typical examples of the progression of hypervascular foci.

\section{Histopathological analysis}

In all eight surgically resected nodules, hypervascular dedifferentiated foci were microscopically identified on hematoxylin \& eosin-stained histological specimens

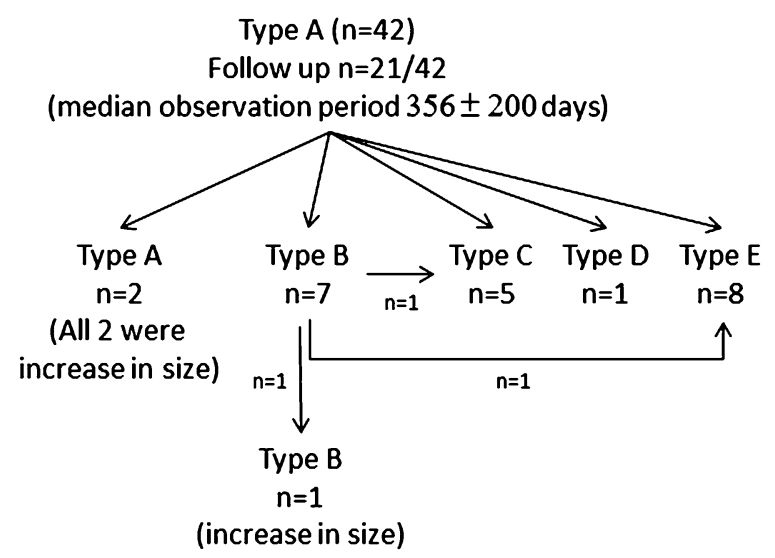

Type $A(n=42)$

Follow up $n=21 / 42$

(median observation period $356 \pm 200$ days)

(increase in size)
Type $B(n=45)$

Follow up $n=16 / 45$

(median observation period $356 \pm 216$ days)

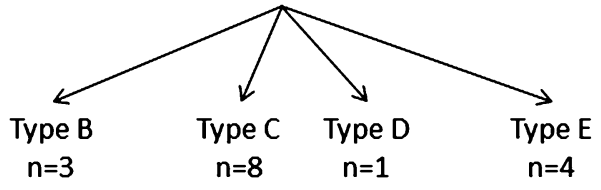

(2 were increase

in size)
Type $C(n=24)$

Follow up $n=1 / 24$

(observation period 393 days)

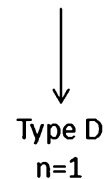

Type D $(n=10)$

Follow up $n=1 / 10$

(observation period 85 days)

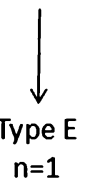

Fig. 4 The progression and hemodynamic changes of an HF in each type during follow-up periods 
(Figs. 7, 8). The de-differentiated foci in the borderline lesion were well-differentiated $\mathrm{HCC}$ in 4 of 8 nodules (Fig. 7) and moderately differentiated $\mathrm{HCC}$ in the remaining 4 (Fig. 8). The background borderline lesion was DN in 5 nodules (Fig. 7) and well-differentiated HCC (corresponding to early HCC) in the remaining 3 nodules (Fig. 8). The differences in the grade of sinusoidal capillarization and MVD of the unpaired arteries, portal venules and hepatic venules between the de-differentiated focus and background borderline lesion are shown in Table 2. The average grade of sinusoidal capillarization and MVD of unpaired arteries in the de-differentiated focus was significantly greater than that in the surrounding borderline lesion $(p<0.05)$. On the other hand, the MVD of hepatic veins in the de-differentiated focus was significantly lower than that in the surrounding borderline lesion $(p=0.01)$. There was no significant difference in MVD of the portal venules between the de-differentiated focus and background borderline nodules. In 4 of 8 nodules, the de-differentiated focus (all were well-differentiated HCCs) demonstrated replacing growth to the surrounding borderline nodule, and abundant sinusoidal communications existed between the two portions (Fig. 7). Three of these 4

a

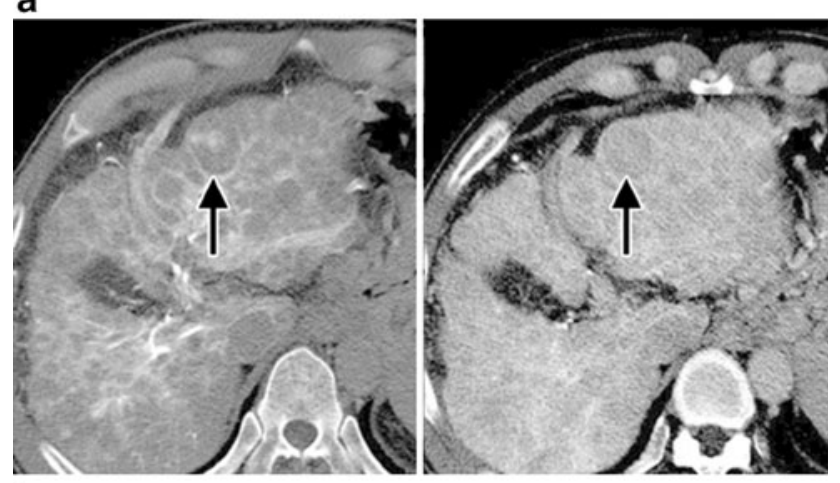

b

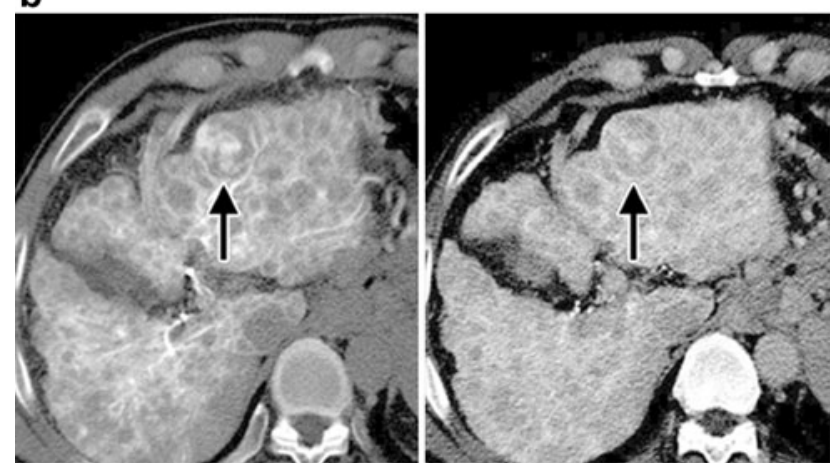

Fig. 5 A 55-year-old man with chronic hepatitis B. a At the first CTHA, an HF in a borderline lesion showed type A hemodynamics (arrows, left early and right late phase). b At the follow-up CTHA obtained after 203 days, the hemodynamics of the HF had been transformed to type B (arrows, left early and right late phase). Both the HF and the entire borderline lesion had slightly enlarged nodules showed type A on CTHA (Fig. 7), and the other one type $\mathrm{B}$. Of the remaining 4 nodules, the de-differentiated focus (all were moderately differentiated HCCs) showed compressive growth to the surrounding borderline lesion and type A, B and D on CTHA, respectively (Fig. 8).

\section{Discussion}

During multi-step hepatocarcinogenesis, an HF appears in a borderline lesion on CTHA, and almost all nodules of this type progress to entirely hypervascular classic HCC within 3 years [8], although the sequential changes in hemodynamics of an HF in this process have not been well clarified. In this study, we verified that the hemodynamic patterns of an HF in a borderline lesion observed on singlelevel dynamic CTHA and/or double-phase CTHA could be classified into four types, namely type A, B, C and D, and they changed in order from type $A$ at the early stage to type $\mathrm{D}$ at more progressed de-differentiation. These hemodynamic changes of the hypervascular foci in hypovascular borderline lesions are very similar to the hemodynamic

a
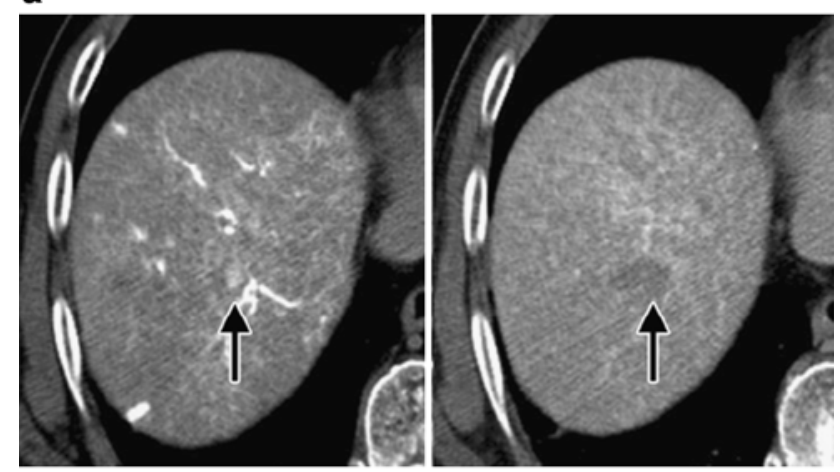

b

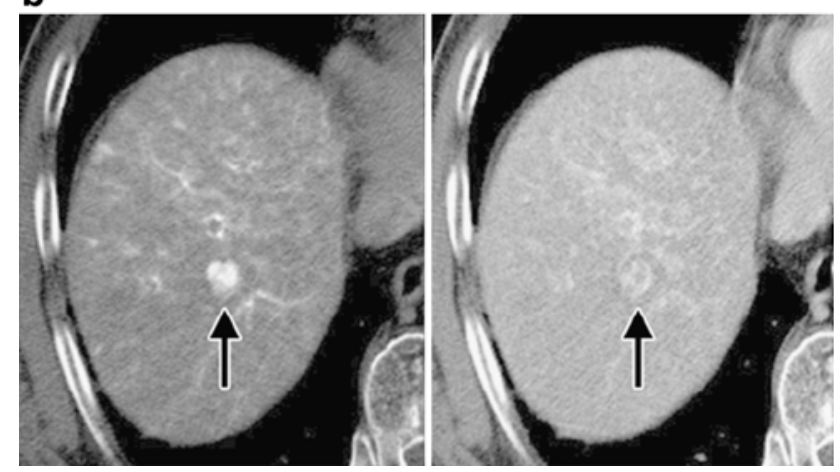

Fig. 6 A 76-year-old man with liver cirrhosis C. a At the first CTHA, an HF in the borderline lesion showed type A hemodynamics (arrows, left: early and right late phase). b At the follow-up CTHA obtained after 307 days, the hemodynamics of the HF had been transformed to type C (arrows, left early and right late phase). The HF had enlarged, while the size of the borderline lesion was unchanged 


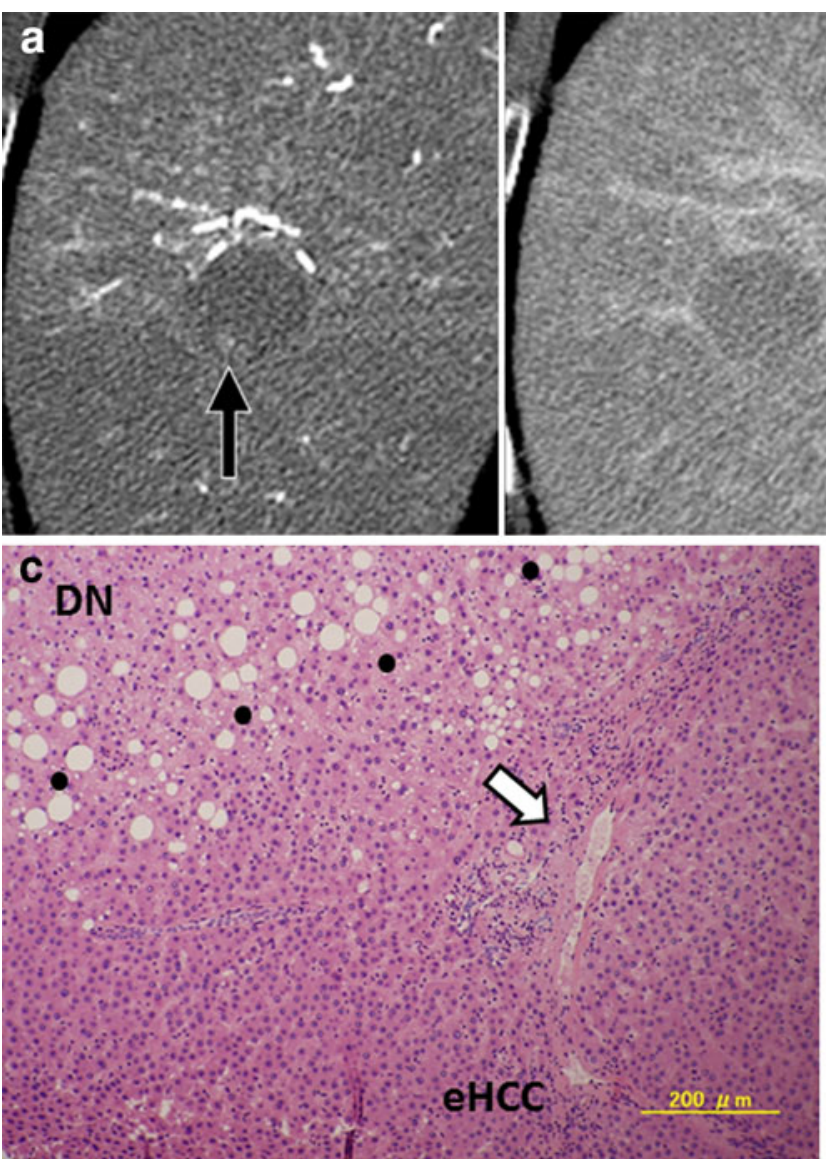

Fig. 7 A 78-year-old man with chronic hepatitis of unknown etiology. a Early phase of CTHA (left) shows an HF (arrow) relative to the surrounding hypovascular borderline lesion (arrowheads). No definite staining was demonstrated in late phase, indicating type A hemodynamics of an HF. b Loupe image of the resected specimen showed a nodule with an indistinct margin (outer dots, borderline between nontumoral surrounding liver and the nodule). The nodule showed a nodule-in-nodule appearance, and the inner nodule showed an early hepatocellular carcinoma (eHCC) focus (inner dots) against the surrounding dysplastic nodule $(D N)$. NT nontumoral surrounding

changes from early $\mathrm{HCC}$ to classic hypervascular $\mathrm{HCC}$ as reported by Kitao et al. [9]. Namely, drainage vessels of HCC change from hepatic veins to hepatic sinusoids and then to portal veins during multistep hepatocarcinogenesis resulting in the sequential changes of peritumoral enhancement from no enhancement in early HCCs (borderline lesions) to definite coronal enhancement in hypervascular classic HCCs [9]. In addition, the sizes of both the entire borderline lesion and HF increased in order from type A to $\mathrm{D}$; this fact also indicated multi-step progression of hepatocarcinogenesis from type A to D.

On the basis of our findings, a mechanism for how the drainage vessels of an HF change during its progression can be postulated (Fig. 9). In type A nodules, the hepatic venules in and surrounding the hypervascular foci decrease but remain sufficient to drain the blood from these
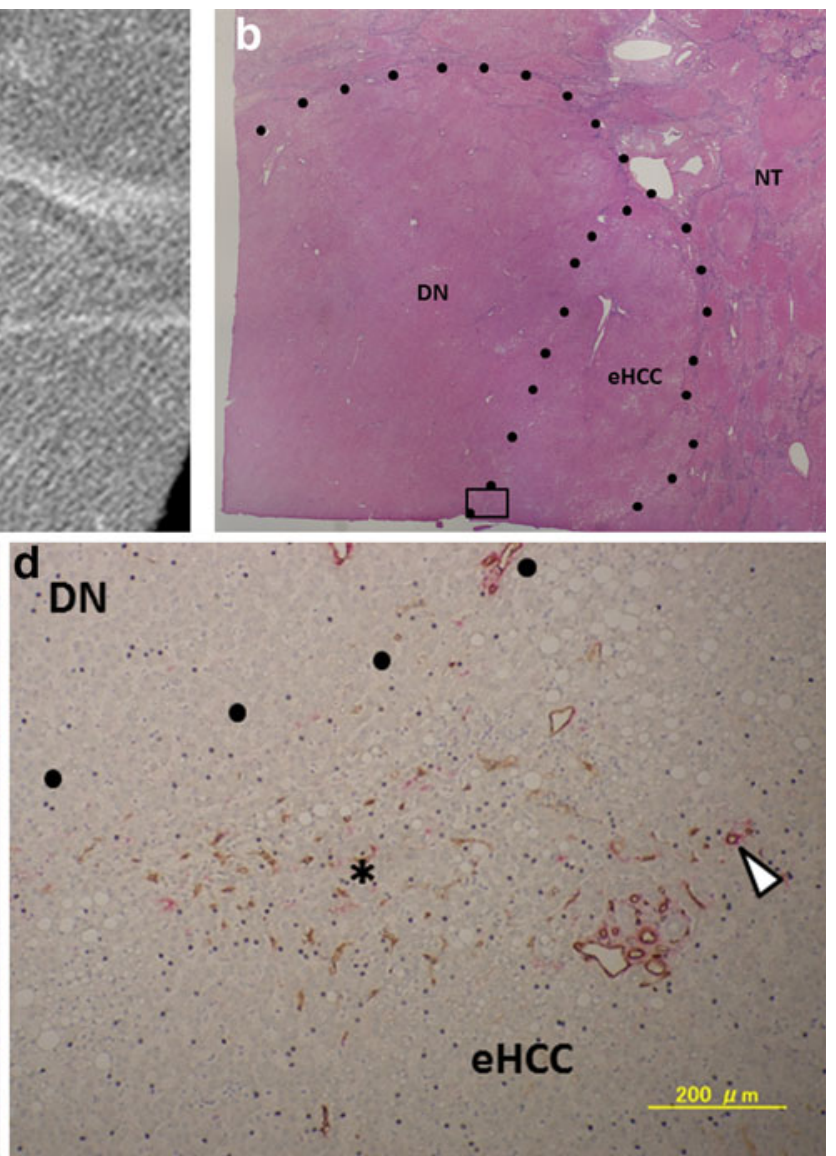

liver. c Microscopic findings of the interface between the inner eHCC focus and DN were very vague and showed replacing growth [high magnification of the rectangular area in $(\mathbf{b}), \mathrm{H} \& \mathrm{E}$ stain $\times 100]$. We could identify stromal invasion of portal tracts in the eHCC focus here and there (arrow). d Double immunohistochemical staining of CD34 (brown) and alpha-smooth muscle actin (red) in DN (left) and the inner eHCC focus (right) demonstrated an increased number of sinusoidal capillarizations and unpaired arteries (arrowhead) in the latter (right)

arterialized foci into these hepatic venules, resulting in an absence of definite prolonged staining in and surrounding the hypervascular foci [9] on the late phase of CTHA. In the presented study, we confirmed a significantly greater number of hepatic venules in the surrounding borderline lesion than in the de-differentiated focus. Then, in accordance with the progression of hypervascular foci, the hepatic venules may decrease from the hypervascular foci as also indicated in this study, and blood from them may drain into the surrounding borderline lesion very gradually, mainly through the communications between blood sinusoids in hypervascular foci and surrounding borderline lesion. In this stage, a prolonged enhancement of the hypervascular foci without definite peri-lesional enhancement can be seen, as shown in type B. Subsequently, in accordance with further increases in the size and 

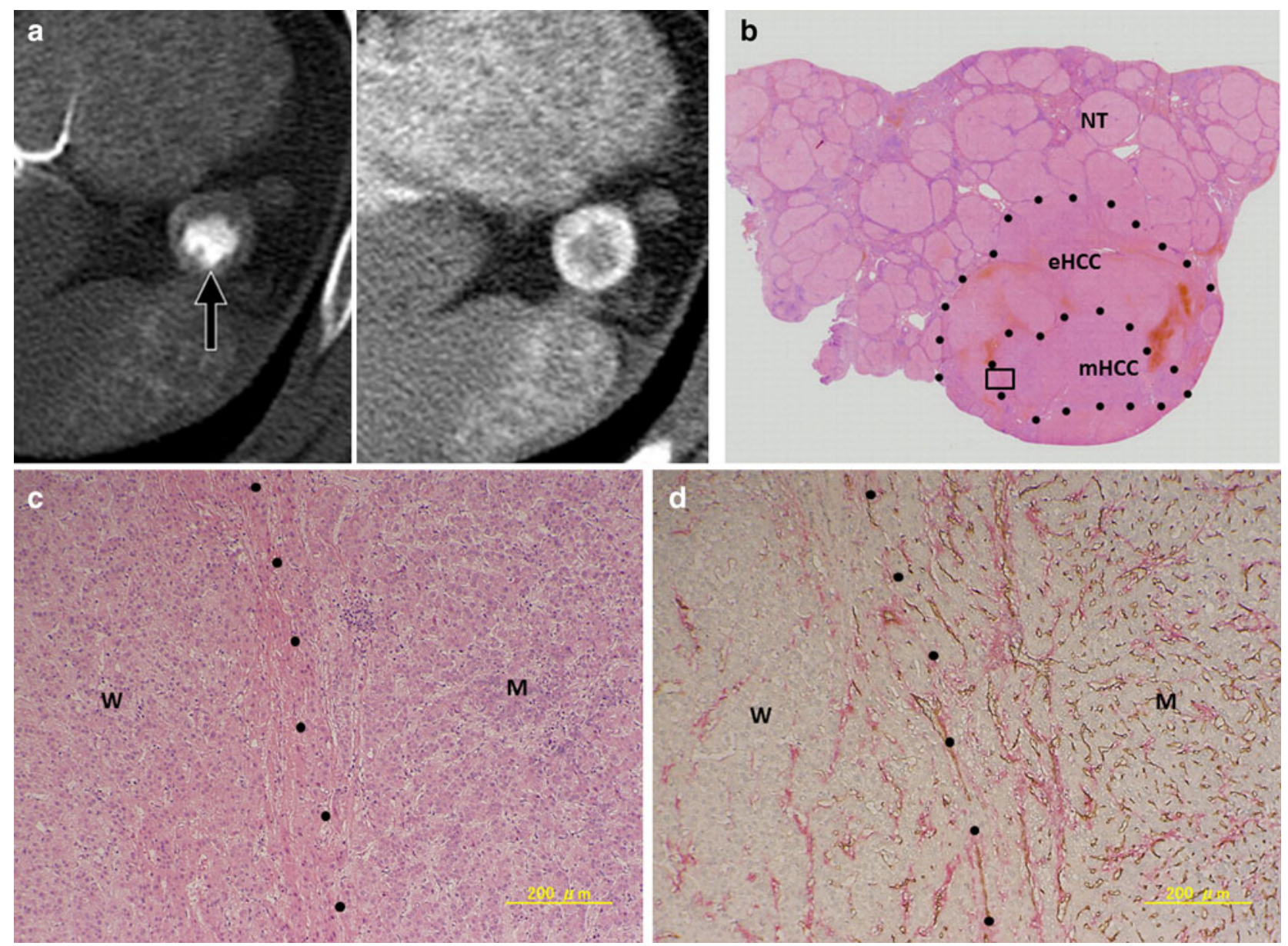

Fig. 8 A 57-year-old man with HCV-related liver cirrhosis. a Early phase of CTHA (left) shows an HF (arrow) relative to the surrounding hypovascular borderline lesion (arrowheads). The entire borderline lesion except for the HF showed diffuse staining on the later phase (right) indicating type D hemodynamics of an HF. b Loupe image of the resected specimen showed a nodule with an indistinct margin (outer dots, borderline between nontumoral surrounding liver and the nodule). The nodule showed a nodule-in-nodule appearance, and the inner nodule showed a moderately differentiated hepatocellular

arterialization of de-differentiated foci, they demonstrate expansive growth to the surrounding borderline lesion. In this stage, the contrast medium from the hypervascular foci drains into the surrounding compressed blood sinusoids in the borderline lesion through the communications between blood sinusoids in hypervascular foci and blood sinusoids and/or portal venules in the surrounding borderline lesion resulting in a thin coronal enhancement. After this stage, the HF grows larger and more expansive against a borderline lesion resulting in more prominent coronal enhancement spreading into the entire borderline lesions as revealed in type $\mathrm{D}$. In addition, the sizes of both the entire borderline lesion and HF increased in order from type A to $\mathrm{D}$, and this fact also indicated multi-step progression from type A to D during hepatocarcinogenesis. carcinoma focus (inner dots, mHCC) against surrounding early HCC (eHCC). NT nontumoral surrounding liver, $m H C C$ moderately differentiated HCC, $e \mathrm{HCC}$ early HCC. c Microscopic findings of the interface between the inner mHCC and eHCC showed compressive growth (high magnification of the rectangular area on (b), H\&E stain $\times 100$ ). d Double immunohistochemical staining of CD34 (brown) and alpha-smooth muscle actin (red) in eHCC (left) and the inner mHCC focus (right) demonstrated an increased number of sinusoidal capillarizations and unpaired arteries in the latter (right)

These changes cannot be well visualized by conventional dynamic contrast imaging studies with CT, MRI or contrast ultrasound as compared with angiography-assisted CT $[13,14]$. Therefore, the clinical application of the results obtained from this study is limited. However, understanding the concept outlined in this study may help understand the basic background of the findings of conventional dynamic blood flow imaging. For example, on dynamic CT or MR imaging, a faint enhancement without washout in a hypovascular borderline lesion may strongly indicate definite malignant transformation of the nodule [13], a tiny hypervascular pseudolesion (arterio-portal shunting) like enhancement can be seen in an early stage HCC, a tiny early enhancement that is prolonged on the late phase may indicate the nodule-in-nodule type of $\mathrm{HCC}$, 
Table 2 Histopathology and intranodular vessels in de-differentiated foci and background borderline lesions

\begin{tabular}{llll}
\hline & $\begin{array}{l}\text { Hypervascular area }(n=8) \\
\text { (inner nodule: de-differentiated region) }\end{array}$ & $\begin{array}{l}\text { Hypovascular area }(n=8) \\
\text { (outer nodule: differentiated region) }\end{array}$ \\
\hline Differentiation (M/W/D)* & $4 / 4 / 0$ & $0 / 3 / 5$ & 0.005 \\
Sinusoidal capillarization (grade) & $3.1 \pm 1.0$ & $1.4 \pm 0.5$ & 0.01 \\
Unpaired arteries [MVD $\left.\left(\mathrm{mm}^{2}\right)\right]$ & $1.2 \pm 1.1$ & $0.2 \pm 0.2$ & 0.02 \\
Portal veins [MVD $\left.\left(\mathrm{mm}^{2}\right)\right]$ & $0.2 \pm 0.2$ & $0.2 \pm 0.1$ & 0.5 \\
Hepatic veins [MVD $\left.\left(\mathrm{mm}^{2}\right)\right]$ & $0.04 \pm 0.7$ & $0.2 \pm 0.2$ & 0.02 \\
\hline
\end{tabular}

Sinusoidal capillarization grade indicated the percentage of CD34 expressing capillarized sinusoid area as follows: $1(0-25 \%), 2$ (26-50 \%), 3 $(51-75 \%)$ and $4(76-100 \%)$

$M$ moderately differentiated hepatocellular carcinoma, $W$ well-differentiated hepatocellular carcinoma (including early hepatocellular carcinoma), $D$ dysplastic nodule, $M V D$ microvessel densities
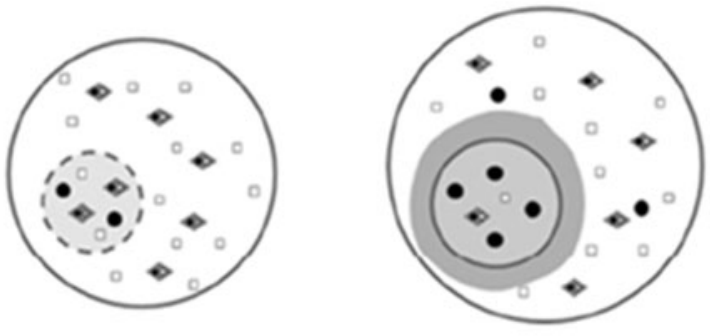

- portal tract

- unpaired artery

hepatic venule

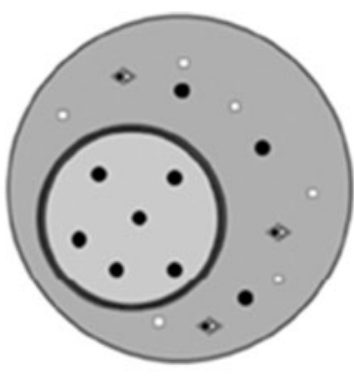

\section{Hypervascular focus (inner nodule)}

Arteries
Portal veins
Hepatic veins
Hypovascular borderlin
Arteries
Portal veins
Hepatic veins
Interface between
inner and outer nodule
Drainage route / area

Radiologic findings

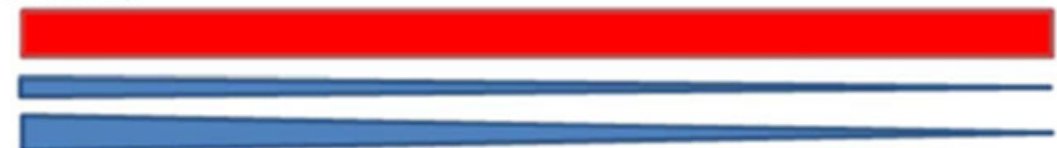

lesion (outer nodule)

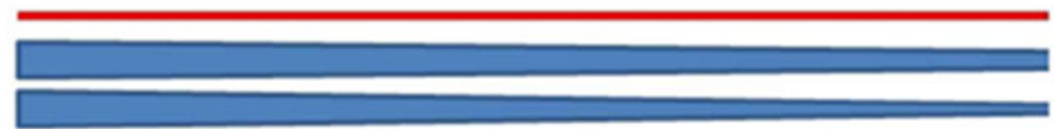

\begin{tabular}{|c|c|c|}
\hline Replacing growth & Compressive growth & Pseudocapsule formation \\
\hline $\begin{array}{l}\text { hepatic veins } \\
\text { in inner nodule }\end{array}$ & $\begin{array}{l}\text { hepatic veins } \\
\text { in surrounding outside of inner } \\
\text { nodule (via sinusoids) }\end{array}$ & $\begin{array}{l}\text { hepatic veins } \\
\text { in entire outer nodule } \\
\text { (via portal veins) }\end{array}$ \\
\hline Type B & Type C & Type D \\
\hline
\end{tabular}

Fig. 9 An HF shows a reduction of portal veins and hepatic veins according to its progression, and the growth pattern of an HF against a surrounding borderline nodule changes from replacing to compressive (with connection of sinusoids) and finally to forming a pseudocapsule (loss of sinusoidal connection). Simultaneously, drainage flow from an HF changes from hepatic veins in an HF to tumor sinusoids in a

surrounding outer borderline nodule via sinusoidal communications between them, and finally to tumor sinusoids in the surrounding borderline nodule via portal veins in the pseudocapsule. Light gray area hypervascular foci, gray area drainage area from hypervascular foci

and iodized oil can obliterate the blood sinusoids of the hypovascular borderline lesion through the drainage route from the hypervascular foci [15].

This study has several limitations. First, it is retrospective in nature, and the follow-up angiography-assisted CT

was carried out at various time intervals according to the timing of transcatheter arterial embolization for HCCs located in different liver segments in each individual. Second, we did not analyze the interobserver correlation because of the straightforwardness of the image 
interpretation. However, it may be needed to verify the adequacy of CTHA classification of an HF in borderline lesions. Third, the histological study was carried out only in a small number of patients because nowadays surgical resection is not performed for these kinds of early stage HCCs. In spite of these important but unavoidable limitations, we think that this study is valuable for the abovementioned reasons.

In conclusion, it was revealed that a hypervascular de-differentiated focus in a hypovascular borderline nodule observed on CTHA showed multistep hemodynamic changes from no prolonged enhancement, faint prolonged enhancement, washout from an $\mathrm{HF}$ with thin coronal enhancement surrounding it, and washout from an HF with diffuse enhancement of the entire borderline lesion. These hemodynamic changes may be due to the progression of arterial neoangiogenesis with a decrease of intralesional hepatic venules.

Acknowledgments We thank Dr. Motoko Sasaki and Dr. Yasunori Sato for valuable pathological suggestions, Dr. Toshihiro Yagi for preparation of surgical specimens, Dr. Masaaki Kitaraha for preparation of medical records, and Mr. John Gelblum for English proofreading.

Conflict of interest The authors declare that they have no conflict of interest.

\section{References}

1. Parkin DM, Bray F, Ferlay J, Pisani P. Global cancer statistics, 2002. CA Cancer J Clin. 2005;55(2):74-108.

2. Wurmbach E, Chen YB, Khitrov G, Zhang W, Roayaie S, Schwartz $\mathrm{M}$, et al. Genome-wide molecular profiles of HCV-induced dysplasia and hepatocellular carcinoma. Hepatology. [Research Support, NIH, Extramural Research Support, Non-US Gov't]. 2007;45(4):938-47.

3. ICGHN, IWP. Pathologic diagnosis of early hepatocellular carcinoma: a report of the international consensus group for hepatocellular neoplasia. Hepatology. 2009;49(2):658-64.

4. Nakamura K, Zen Y, Sato Y, Kozaka K, Matsui O, Harada K, et al. Vascular endothelial growth factor, its receptor Flk-1, and hypoxia inducible factor-1 alpha are involved in malignant transformation in dysplastic nodules of the liver. Hum Pathol. 2007;38(10):1532-46.
5. Arakawa M, Kage M, Sugihara S, Nakashima T, Suenaga M, Okuda K. Emergence of malignant lesions within an adenomatous hyperplastic nodule in a cirrhotic liver. Observations in five cases. Gastroenterology. [Case Reports Research Support, NonUS Gov't]. 1986;91(1):198-208.

6. Matsui O, Kadoya M, Kameyama T, Yoshikawa J, Takashima T, Nakanuma $\mathrm{Y}$, et al. Benign and malignant nodules in cirrhotic livers: distinction based on blood supply. Radiology. 1991; 178(2):493-7.

7. Hayashi M, Matsui O, Ueda K, Kawamori Y, Kadoya M, Yoshikawa $\mathrm{J}$, et al. Correlation between the blood supply and grade of malignancy of hepatocellular nodules associated with liver cirrhosis: evaluation by CT during intraarterial injection of contrast medium. AJR. 1999;172(4):969-76.

8. Hayashi M, Matsui O, Ueda K, Kawamori Y, Gabata T, Kadoya M. Progression to hypervascular hepatocellular carcinoma: correlation with intranodular blood supply evaluated with CT during intraarterial injection of contrast material. Radiology. 2002;225 (1):143-9.

9. Kitao A, Zen Y, Matsui O, Gabata T, Nakanuma Y. Hepatocarcinogenesis: multistep changes of drainage vessels at CT during arterial portography and hepatic arteriography-radiologic-pathologic correlation. Radiology. 2009;252(2):605-14.

10. Ueda K, Matsui O, Kawamori Y, Nakanuma Y, Kadoya M, Yoshikawa J, et al. Hypervascular hepatocellular carcinoma: evaluation of hemodynamics with dynamic CT during hepatic arteriography. Radiology. 1998;206(1):161-6.

11. Murakami T, Takamura M, Kim T, Hori M, Federle MP, Onishi $\mathrm{H}$, et al. Double phase CT during hepatic arteriography for diagnosis of hepatocellular carcinoma. Eur J Radiol. 2005;54(2): 246-52.

12. Inoue E, Fujita M, Hosomi N, Sawai Y, Hashimoto T, Kuroda C, et al. Double phase CT arteriography of the whole liver in the evaluation of hepatic tumors. J Comput Assist Tomogr. 1998; 22(1):64-8.

13. Shinmura R, Matsui O, Kadoya M, Kobayashi S, Terayama N, Sanada $J$, et al. Detection of hypervascular malignant foci in borderline lesions of hepatocellular carcinoma: comparison of dynamic multi-detector row $\mathrm{CT}$, dynamic MR imaging and superparamagnetic iron oxide-enhanced MR imaging. Eur Radiol. 2008;18(9):1918-24.

14. Pugacheva O, Matsui O, Kozaka K, Minami T, Ryu Y, Koda W, et al. Detection of small hypervascular hepatocellular carcinomas by EASL criteria: Comparison with double-phase CT during hepatic arteriography. Eur J Radiol. 2011;80(3):e201-6.

15. Miyayama S, Matsui O, Yamashiro M, Ryu Y, Takata H, Takeda $\mathrm{T}$, et al. Iodized oil accumulation in the hypovascular tumor portion of early-stage hepatocellular carcinoma after ultraselective transcatheter arterial chemoembolization. Hepatol Int. 2007;1(4):451-9. 